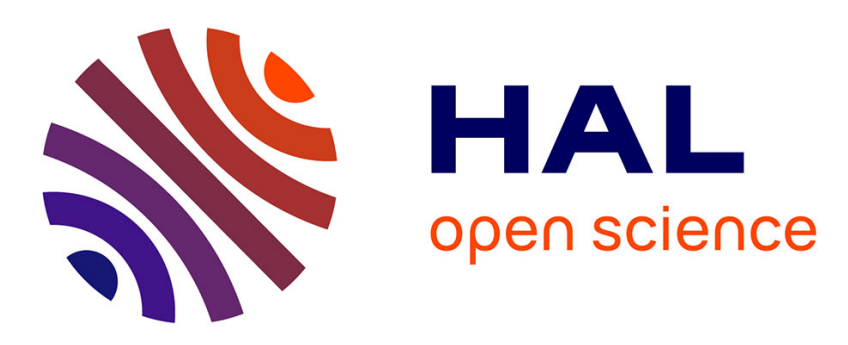

\title{
Glass-LikeMembrane Protein Diffusion in a Crowded Membrane
}

Ignacio Munguira, Ignacio Casuso, Hirohide Takahashi, Felix Rico, Mohamed Chami, Simon Scheuring

\section{- To cite this version:}

Ignacio Munguira, Ignacio Casuso, Hirohide Takahashi, Felix Rico, Mohamed Chami, et al.. Glass-LikeMembrane Protein Diffusion in a Crowded Membrane. ACS Nano, 2016, 10.1021/acsnano.5b07595 . inserm-01285787

\section{HAL Id: inserm-01285787 https://www.hal.inserm.fr/inserm-01285787}

Submitted on 9 Mar 2016

HAL is a multi-disciplinary open access archive for the deposit and dissemination of scientific research documents, whether they are published or not. The documents may come from teaching and research institutions in France or abroad, or from public or private research centers.
L'archive ouverte pluridisciplinaire HAL, est destinée au dépôt et à la diffusion de documents scientifiques de niveau recherche, publiés ou non, émanant des établissements d'enseignement et de recherche français ou étrangers, des laboratoires publics ou privés. 


\title{
Glass-LikeMembrane Protein Diffusion in a Crowded Membrane
}

Ignacio L.B. Munguira ${ }^{1 \#}$, Ignacio Casuso ${ }^{1 \#}$, Hirohide Takahashi ${ }^{1}$, Felix Rico ${ }^{1}$, Atsushi Miyagi ${ }^{1}$, Mohamed Chami ${ }^{2} \&$ Simon Scheuring ${ }^{1}$ *

${ }^{1}$ U1006 INSERM, Université Aix-Marseille, Parc Scientifique et Technologique de Luminy, 163 avenue de Luminy, 13009 Marseille, France

${ }^{2}$ Center for Cellular Imaging and NanoAnalytics, Biozentrum, University of Basel, Mattenstrasse 26, $\mathrm{CH}-$ 4058 Basel, Switzerland

\author{
\#These authors have equally contributed to the work \\ ${ }^{*}$ Correspondence should be addressed to S. Scheuring \\ Email: simon.scheuring@inserm.fr \\ Tel.: ++33-4-91828777, Fax: ++33-4-91828701
}

Many functions of the plasma membrane depend critically on its structure and dynamics. Observation of anomalous diffusion in vivo and in vitro using fluorescence microscopy and single particle tracking has advanced our concept of the membrane froma homogeneous fluid bilayer with freely diffusing proteinsto a highly organized crowded and clustered mosaicof lipidsand proteins. Unfortunately, anomalous diffusion could not be related to local molecular details given the lack of direct and unlabeled molecular observation capabilities. Here, we use high-speed atomic force microscopy and a novel analysis methodology to analyze the pore forming protein lysenin in a highly crowded environment and document coexistence of several diffusion regimes within one membrane. We show the formation of local glassy phases, where proteins are trapped in neighbor-formed cages for time scales up to 10 seconds, which had not been previously experimentally reported for biological membranes. Furthermore, around solid-like patches and immobile molecules a slower glass phase is detected leading to protein trapping and creating a perimeter of decreased membrane diffusion.

Keywords: Anomalous Diffusion / Membrane Dynamics / Membrane Domains / High-Speed Atomic Force Microscopy / Single Molecule / Glass-Glass Transition

\section{Graphical Table of Contents}

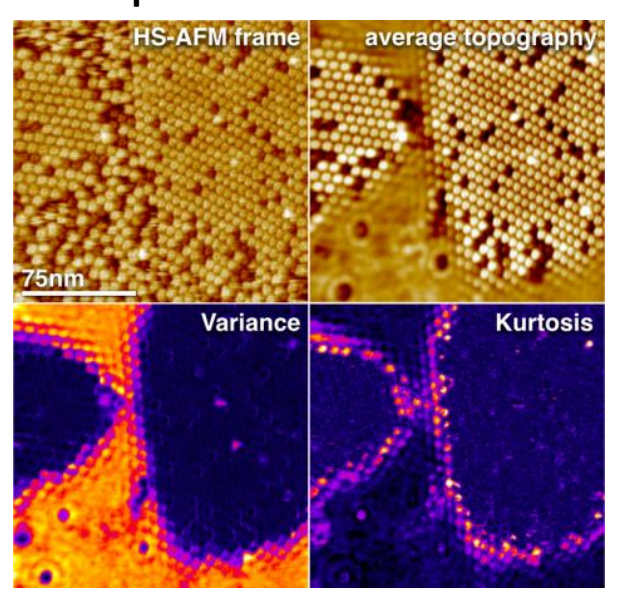


1 Biological membranes are formed by lipids and proteins (and associated sugars). The 2 composition, relative concentration and density of lipids and proteins define their 3 aggregation and diffusion, ${ }^{1}$ which in turn regulate membrane protein distribution and 4 function. ${ }^{2,3}$ In the recent years, evidence has been accumulated that biological 5 membranes are complex and organized, featuring local aggregations of lipids and 6 proteins (rafts) ${ }^{1,4}$ and protein species- and localization-dependent diffusion 7 properties, ${ }^{5}{ }^{6}$ which regulate the interaction of the membrane components and 8 modulate the biomolecular processes taking place in the membrane.

9 Diffusion can be Brownian, i.e. the mean-square displacement (MSD) scales linearly 10 with the time of observation. However, the observed diffusion trajectories of lipids and 11 proteins in biological membranes often deviate from Brownian behavior. Indeed, it is 12 observed that MSD of a membrane componenthas nolinearrelationship with observation time: these types of anomalous diffusions are termed superdiffusion and subdiffusionwhen the object goes farther or stays closer to its initial position, respectively, compared to a Brownian movement. Such intricate and diverse diffusion properties at the cell membrane regulate biomolecular traffic and thus molecular encounters and function. We are just at the beginning of understanding membrane architecture and dynamics. ${ }^{7-9}$

Lysenin $^{10,11}$ is a $33 \mathrm{kDa}$ protein extracted from the coleomic fluid of the earthworm Eiseniafetida. It belongs to the family of Pore-Forming Toxins (PFT), and its mechanism of action is common to PFTand is described in six steps: ${ }^{12}$ Secretion, binding to the target membrane, two-dimensional diffusion,oligomerisation, assemblyin hexagonal close pack,and pre-poreto pore transformation. ${ }^{13}$ Besides Lysenin's affinity for Sphingomyelin (SM), membrane-embedded cholesterol (Chol) has been shown to facilitate the formation of Lysenin oligomers. Lysenin offers hence an excellent system for studying fundamentals of membrane diffusion and aggregation allowing it to interact with mixed SM/Chol bilayers at variable local density. ${ }^{14}$

The general approach to study membrane protein diffusion is fluorescence microscopy combined with single molecule tracking. Fluorescence microscopy allows studying the diffusion of fluorescence-tagged molecules with high temporal resolution and under physiological conditions. ${ }^{6,15,16}$ Unfortunately, fluorescence microscopy comes with some shortcomings: (i) Only tagged molecules can be studied and are visible as long as they are not bleached, and (ii) the lateral and the spatial resolution arelimited to optical diffraction $\sim 200 \mathrm{~nm}$ (distance between two objects that can be resolved in real space at the same time) andto fitting of the point-spread function $20 \mathrm{~nm}$ (typical precision of localization). Furthermore, only one single molecule can be tracked keeping the environment non-fluorescent in the color of the observed tag is prerequisite for the localization of the molecule of interest.

High-Speed Atomic Force Microscopy (HS-AFM) ${ }^{17}$ offers a novel view of membrane protein architecture and dynamics. ${ }^{18}$ It contours proteins with about $\sim 1 \mathrm{~nm}$ lateral and $\sim$.1nm vertical resolution in buffer at ambient temperature and pressure, and atsubsecondrate. Hence, HS-AFM does not only visualize the movement of unlabeled proteins, but it images all molecules within the membrane. Given this novel and unique feat, HS-AFM allows correlating diffusion to the molecular environment. 


\section{RESULTS/ DISCUSSION}

3

4

Here, we use HS-AFM to determine the effect of local molecular crowdingon the diffusion behavior of lysenin on supported lipid bilayers (SLB) constituted of SM/Chol 1:1 (Figure 1a, Supplementary Movie 1). As previouslyreported, ${ }^{13}$ the molecules in pre-pore and pore states can be distinguished based on their membrane protrusion height. Both classes of molecules associate and diffuse next to each other as pre-pores inserts into the first leaflet and pores into both leaflets of the membrane. The resolution and signal-to-noise ratio in the HS-AFM movie allows almost each lysenin ringto be clearly depicted over the entire movie (Figure 1a, left 4 panels). In a time average over the entire movie (Figure 1a, right panel) areas of different morphology and dynamics are readily distinguished: In certain areas proteins form stable lattices and are well resolved after averaging. In contrast, in other areas, no structural features are recognizable reporting high dynamics in these locations. In agreement, the standard deviation (SD) map of the pixel height values over the entire movie duration (Figure 1b, left panel) displayed low values (purple) in the hexagonally packed areas, while the SD was high (yellow) in the diffusive areas. Interestingly, at the interface between these domains, well-defined circular patterns were detected, corresponding to lysenin localizations, where the SD-map displayed low to high SD values(Figure $\mathbf{1 b}$, arrowheads). This indicated that molecules at interfaces revealed association times of varying duration from long (well-preserved topography and low SD) to short (undefined topography and high SD). Some of the pores,about $0.5 \%$ of all molecules got stuck by interactions with the mica supportand served us as proof that the vast majority of the molecules had motional freedom (Figure 1b, dashed squares, Figure 1c, Supplementary Movie 2). Furthermore, these molecules mimic anchored proteinpickets, ${ }^{19}$ and confirm experimentally that immobile molecules can strongly influence the diffusion of its annular environment (Figure 1c):The immobile molecule creates new borders that resemble structurally and dynamically the borders of the crystalline domains.

We analyzed the local dynamics of the proteins, using an approach inspired by Fluorescence Correlation Spectroscopy (FCS). ${ }^{20}$ Since in an AFM measurement, the pixel intensity is the molecular height, the pixel value is directly related to the presence of a molecule under the tip. Hence, the time that a pixel keeps a certain value reports directly about the residence time of a protein and a value change reports thediffusion 'under' that pixel. A section kymograph (Figure 1d, along the dashed line in Figure 1a) illustrates the power of the approach: Pixels constituting the crystalline areas display constant height values as a function of time (Figure 1d, top), while pixels that are part of the diffusive areas presents rapid changes (Figure 1d, bottom). At the border pixels are occupied with stable molecules for intermediate lag-times (seconds to minutes) in alternation with rapid molecular redistribution (Figure 1d, middle).

In order to analyze the diffusion of molecules 'under' each pixel, we calculated thedifference of each pixel value $\left(z_{i}\right)$ at time $t$ with respect to a later moment $t+\tau$ $\left(\Delta z_{i}(t, \tau)=z_{i}(t+\tau)-z_{i}(t)\right)$. As a result, histogramsof the height changes $\Delta z_{i}(\tau)$ of 
1 each pixel $(i)$ and varying lag-time $(\tau)$ were obtained. Such histograms, also termed 2 'van Hove distributions', are commonly used in colloidal sciences to extract diffusion 3 parameters. ${ }^{21}$ From each van Hove distribution on each pixel, the variance $V(\tau)$ is extracted, analogous to the MSD in single molecule tracking, ${ }^{22}$ following

$$
V(\tau)=\frac{1}{n} \sum_{1}^{n}\left(\Delta z_{i}(\tau)-m_{i}(\tau)\right)^{2}
$$

wheren is the number of frames of each pixel $i$, and $m_{i}$ the pixel mean value. Using this approach, variance maps for varying $\tau$ are generated (Figure 2a). As expected, the variance increases with the $\tau$ in all the domains, though much less in the crystalline areas. Whatever the intensity of dynamics, as long as the behavior is Brownian then the van Hove distribution isGaussian. In contrast, non-Brownian dynamics, as found for example in glasses, give rise to non-Gaussian van Hove distributions. This is because particles in glasses are constraint and transiently caged by the dense packing and interactions with neighboring particles, but undergo rare large displacements due to cage rearrangements. To characterize such complex dynamics the Kurtosis Kof the distribution on each pixel is calculated, following

$$
K(\tau)=\frac{\left.\frac{1}{n} \sum_{1}^{n}\left(z_{i}(t+\tau)-z_{i}(t)\right)-m_{i}\right)^{4}}{\left.\left(\frac{1}{n} \sum_{1}^{n}\left(z_{i}(t+\tau)-z_{i}(t)\right)-m_{i}\right)^{2}\right)^{2}}
$$

When $K=3$, then the distribution in the van Hove plot is Gaussian and the underlying motion is Brownian. Deviations from $K=3$ are associated with anomalous diffusion, and typically $K>3$ are signature of the cooperative behavior in glasses. ${ }^{23}$

Plotting Kon each pixel as a function of varying $\tau$, areas of non-Brownian dynamics are highlighted (Figure $\mathbf{2 b}$ ). Within the fluid areas, we find subregions, upper left corner, which display a Kurtosis $\sim 3$ at all $\tau$,corresponding to free diffusion. However, most of the fluid domains revealed Kurtosis significantly $>3$ at short lag-times, and $\sim 3$ when analyzed over longer $\tau>8 \mathrm{~s}$, corresponding to the average trapping time of particlesin this glass phase.Beyond this $\tau$ the glass behaves like a Brownian fluid. The interfacesbetween fluid and crystalline domains displayed high Kurtosis values $>4$ even overextended lag-times indicating that molecules are trapped for varying durations and eventually up to minutes. The crystalline domains despite their low dynamics displayed $K \sim 3$, and correspondtherefore statistically to an area of Brownian diffusion.

To better understand the relationship between the structure and dynamics of these domains, ${ }^{24}$ we undertook two types of analysis:First, for a more detailed comprehension of the crystalline domains we performed negative stain electron microscopy (EM) (Supplementary Figure S1a) combined with single particle analysis (Supplementary Figure S1b), and cryo-EM (Supplementary Figure S1c) combined with electron crystallography (Supplementary Figure S1d) of lysenin as individual molecules and in the crystalline arrangement, respectively. Both approaches depicted lyseninas a nonamericpore with two concentric density rings.The 9-fold symmetric molecule assembles with $p 3$-symmetry in the 'hexagonal packing' with $a=b=12 \mathrm{~nm}$ and $\gamma=120^{\circ}$, in agreement with HS-AFM (Figure 1a).In such an arrangement onelysenin ring occupies a membrane area $A=2 \cdot(\sqrt{3} / 4 \cdot a \cdot b)$ of $125 \mathrm{~nm}^{2}$ corresponding to an area fraction $(\phi)$ 
1 of 0.91 . Second, for a more detailed comprehension of the fluid domains, we 2 performed Delaunay triangulationand Voronoi tessellation 3 (Supplementary Figure S2a). ${ }^{25}$ In the case of a 2D-lattice, each molecule has 6 nearest 4 neighbors and the Voronoi cells are hexagons with $125 \mathrm{~nm}^{2}$ area. In the fluid domain, 5 the average distance between molecules is $16.5 \pm 3.5 \mathrm{~nm}$, with about 5.5 nearest 6 neighbors, and pores occupy areas up to $250 \mathrm{~nm}^{2}$, an approximate area fraction of 0.45 without short- or long-range order (Supplementary Figure S2b).Furthermore, the shape factor $\xi$ of the Voronoi cells have been analyzed: Each cell is characterized by a shape factor $\xi=\left(C^{2} / 4 \pi A\right)$, where $C$ is the perimeter and $A$ the area of the Voronoi cell. In the range $\phi \approx 0.60-0.71$ a bimodal distribution of the shape factor is observed, meaning that molecules in the same density range have different number of neighbors, characteristic of phase transitions (Supplementary Figure S3). ${ }^{26}$

Having in hand methodologies to characterize diffusion properties (Figure 2) and local protein density (Supplementary Figure S2a) 'under' each pixel, we had competence to determine how diffusion properties scale and change as function of local membrane structure.Plotting the variance of all pixels in the movie as a function of the Voronoi cell area that comprises each pixel and as a function of varying $\tau$, a segregation of the data occurred (Figure 3a). A large number of data points gather at a Voronoi cell size of $\sim 125 \mathrm{~nm}^{2}$ and small variance that remains unchanged with increasing lag-time; these pixels are part of the crystalline area. In contrast, pixels in areas of low protein density and Voronoi cell size $>200 \mathrm{~nm}^{2}$ show high variance that further increases with $\tau$. The Kurtosis of the crystalline area, Voronoi cell sizes of $\sim 125 \mathrm{~nm}^{2}$, is stable at a value of $\sim 3$, Brownian rattling(Figure $\mathbf{3 b}$ ). However, pixels that locate in the fluid areas with low protein density and Voronoi cell area $>200 \mathrm{~nm}^{2}$ depict wide spread Kurtosis values with many significantly $>3$ at short $\tau$.

In analogy to typical MSD vs lag-time plots from single particle tracking, we plotted variance vs lag-time.Our approach has the advantage that proteins can be grouped together as a function of the local density in which theyevolve.In general, the variance increaseswithlower local protein density (larger Voronoi cell). The slope of the variance as a function of $\tau$ is somewhat steeper at $240 \mathrm{~nm}^{2} /$ molecule comparedto areas at $125 \mathrm{~nm}^{2} /$ molecule at short $\tau$, flattening at longer $\tau$. However, at intermediate protein density of $\sim 185 \mathrm{~nm}^{2} /$ molecule the slope is increased, especially for longer $\tau$, indicating particular diffusion of molecules at this density (Figure 3c, left). When plotting the variance vsVoronoi cell area for different $\tau$, we observe a biphasic, maybe triphasic, behavior: Low variance for the crystalline areas with $125 \mathrm{~nm}^{2}$ to $165 \mathrm{~nm}^{2}$ per molecule, high variance for fluid areas with $200 \mathrm{~nm}^{2}$ to $250 \mathrm{~nm}^{2}$ per molecule, and a steep variance increase for intermediate molecular density regions with $165 \mathrm{~nm}^{2}$ to $200 \mathrm{~nm}^{2}$ per molecule. Around Voronoi areas of $185 \mathrm{~nm}^{2}$ a weak variance plateauat short $\tau$, is found (Figure 3c, right).

Plotting the Kurtosis as a function of lag-time for the different Voronoi cells revealedthree different populations of Brownian and non-Brownian dynamics (Figure 3d, left): Densely packed molecules have Kurtosis $\sim 3$, almost independent of $\tau$. When the localprotein density is lower than $165 \mathrm{~nm}^{2}$ per molecule, thenthe Kurtosis raises abruptly to a completely non-Brownian regime.A third population is found when the protein density loosens further with Voronoi cells of $200 \mathrm{~nm}^{2}$ and larger: In this 
1 regime, the Kurtosis is significantly non-Brownian at $\tau<10 \mathrm{~s}$, above this lag-time 2 diffusion is Brownian. 10s seems to be the average trapping time of lyseninin the 3 glassy-fluid phase. This striking triphasic behavior and its transitions are best visualized 4 when plotting the Kurtosis as a function of Voronoi cell area (Figure 3d, right): 5 Molecules evolving in a density regime of $165 \mathrm{~nm}^{2}$ to $200 \mathrm{~nm}^{2}$ membrane area (peaking 6 at $185 \mathrm{~nm}^{2}$ ) display non-Brownian diffusion characteristics basically independent of the

7 time span. These characteristics around $\phi \sim 0.61$ arein good agreement with theory and 8 simulation of2D-glasses. ${ }^{27,28}$

HS-AFM features the advantage to visualize (i) non-labeled molecules and (ii) not only single moleculesbut all molecules in the membrane. An approach inspired by FCS is used to detect dynamics 'under' each pixel with nanometer resolution, of particular importance in crowded systems.Qualitatively similar results were obtained fromautomated single particle tracking(Supplementary Figure S4). However considerable problems occur when tracking densely packed molecules, where the motion of the molecules is comparable to the inter-particle distance. Furthermore, a single local densitycannot be attributed to a molecule trajectory as the molecule may diffuse through heterogeneous domains. In contrast, the pixel-by-pixel analysisapproach used here(Figure $\mathbf{3}$ ) provides the possibility to correlate density and diffusion.

\section{CONCLUSIONS}

In summary, the membrane contains four phases with different diffusion dynamics: At $\phi$ ranging from 0.91 to 0.68 (solid phase), the proteins are essentially crystalline and rattle around their position with Brownian dynamics.At borders of the solid phase, the proteins evolve at $\phi$ between 0.68 and 0.56 (sliding glass) and are caged up to minutes. Morphologically, this glassy area resembles a 'sliding puzzle' where moving complexes occupy defined positions. At $\phi 0.56$ to 0.45 (fluid glass) diffusion is fluid yet molecules are caged at shorter time periods $<10$ seconds. Calculating SD maps running over 10 s of movie acquisition revealed the presence of spatially correlated dynamics represented by SDfluctuation waves (Supplementary Movie 4). This glassy phase is characterized by lack of short-range order.At $\phi$ below 0.45 (liquid phase) proteins diffuse freely. Such a coexistence of several glasseshas been described in colloidal systems. ${ }^{29,30}$

In a crowded mosaic biological membrane, molecules diffusein an environment with $\phi \sim 0.5$ containing stable domains. ${ }^{1,}$ Furthermore, molecular heterogeneity favors the occurrence of glass dynamics. ${ }^{24}$ Specialized membranes,e.g.photosynthetic membranes ${ }^{31}$ or retinal disk membranes ${ }^{32,33}$ may be even more crowdedand comply to cooperative rearrangements during their functional tasks.

HS-AFM allowscorrelating structure with diffusion behavior, and glassy diffusion is only detectable when both movement and environment are simultaneously assessed. Therefore, biologists may have missed glass-like diffusion in crowded membranes ${ }^{34}$ due to the technical limitation of only tracking single molecules. Given the crowdedness of cellular membranes, we hypothesize that glassy dynamics might be a 
METHODS/EXPERIMENTAL

3

4

\section{Protein purification}

A cDNA fragment, coding for Lysenin (GenBank: BAA21518.1,GenScript, USA) and cloned into pET28a vector at BamHI and Hind III, and this vector transformedinto BL21(DE3) strain (New England BioLabs France, Evry, France). Thetransformed cells were inoculated into 1 liter of LB medium containing $100 \mu \mathrm{g} / \mathrm{ml}$ kanamycin sulfate, and incubated at $37^{\circ} \mathrm{C}$ while shaking at $200 \mathrm{rpm}$ until the $O D_{600}$ value reached 0.6.For induction of Lysenin expression, isopropyl $\beta$-D-1-thiogalactopyranoside (IPTG) was added at final concentration of $0.5 \mathrm{mM}$, and cells shaken overnight at $20^{\circ} \mathrm{C}$ and $200 \mathrm{rpm}$. The bacteria were collected by centrifugation at $2000 \mathrm{~g}$ for 10 minutes, and disrupted with 3 probe sonicator intervals of 15 seconds sonication and 30 seconds ice cooling each. The resulting suspension was shaken at $4^{\circ} \mathrm{C}$ for 30 minutes in Triton X100 at $0.1 \%$ and RNase/DNaseat $10 \mu \mathrm{g} / \mathrm{ml}$ concentrations. The mixture was centrifuged at $10^{4} \mathrm{~g}$ for 30 minutes. The supernatant (volume: $9 \mathrm{ml}$ ) was collected and mixed with $1 \mathrm{ml}$ of metal chelating resin, chelating sepharose Fast flow (GE Healthcare France), composed of chelating cobalt in $100 \mathrm{mMNaCl}$, Hepes- $\mathrm{NaOH}$, pH 7.5.Lysenin binding was performed by 1 hour incubation at $4^{\circ} \mathrm{C}$ with gentle shaking, and the resulting resin centrifuged at $100 \mathrm{~g}$ for 1 minute to discard the supernatant. The resin was washed with fresh $14 \mathrm{ml}$ of $100 \mathrm{mMNaCl}, 100 \mathrm{mM}$ imidazole-HCl, Hepes- $\mathrm{NaOH}$, $\mathrm{pH} 7.5$ by 3 centrifuge/washing cycles. Lysenin was eluted in $1 \mathrm{ml}$ of $100 \mathrm{mMNaCl}$, $250 \mathrm{mM}$ imidazole- $\mathrm{HCl}$, Hepes- $\mathrm{NaOH}, \mathrm{pH}$ 7.5. To eliminate the imidazole, the eluate was dialyzed against 1 liter of $100 \mathrm{mMNaCl}$, Hepes- $\mathrm{NaOH}, \mathrm{pH}$ 7.5. The resulting protein sample was directly used for the HS-AFM experiment.

\section{Samplepreparation for High-speed atomic force microscopy (HS-AFM) observation}

Egg Sphingomyelin(SM) andCholesterol (chol) (Avanti Polar Lipids, Alabama, USA)were used to form giant unilamellar vesicles (GUVs) at a molar ratio SM:Chol 1:1 through electroswelling. ${ }^{35}$ of each lipid10 $\mu \mathrm{l}$ at $3 \mathrm{mMdissolved}$ in chloroform: methanol 3:1 were deposited in two glass plates coated with indium tin oxide with 70-100 $\Omega$ resistivity(Sigma-Aldrich) and placed 60 minutes in the desiccator for complete solvent evaporation. A U-shaped rubber piece of $\sim 1 \mathrm{~mm}$ thickness was sandwiched between the two indium tin oxide coated slides. The so-formed chamber was filled with $\sim 400 \mu$ lof $200 \mathrm{mM}$ sucrose solution and exposed to $1.5 \mathrm{~V}$ sinusoidal $10 \mathrm{~Hz}$ AC current for 3 hours followed bysquared $5 \mathrm{~Hz} \mathrm{AC}$ current for 15 minutes, at $55^{\circ} \mathrm{C}$. GUVs were harvested from the chamber. To form the supported lipid bilayers (SLBs) for HS-AFM, $1 \mu \mathrm{l}$ of GUV solution was placed ona $1.5 \mathrm{~mm}$-diameterfreshly cleaved mica disk covered with $1 \mu \mathrm{l}$ of phosphate buffer saline (PBS) and incubated for 30 minutes.To remove lipid that was not firmly attached the SLB was intensely rinsed with PBS. Once the bilayer was formed, $1 \mu$ of purified lysenin was incubated for 15 minutes. Excess of protein was again rinsed with PBS.

\section{High-speed atomic force microscopy (HS-AFM)}


1 HS-AFM movies were acquired with an Ando-type setup ${ }^{17}$ equipped with a super 2 luminescent diode (emission wavelength: $750 \mathrm{~nm}$; EXS 7505-B001, Exalos, Schlieren, 3 Switzerland) and a digital high-speed lock-in Amplifier (Hinstra, Transcommers, 4 Budapest, Hungary). ${ }^{36} 8 \mu \mathrm{m}$-long cantilevers with spring constant $k=0.15 \mathrm{Nm}^{-1}$, 5 resonance frequency $f_{(r)}=500-700 \mathrm{kHz}$ and quality factor $Q \approx 1.5$ in liquid (USC-1.2, 6 NanoWorld, Neuchâtel, Switzerland), featuring an electron beam deposition (EBD) tip, 7 were used.For high-resolution imaging the tip was sharpened by helium plasma 8 etching using a plasma cleaner (Diener electronic, Ebhausen, Germany), resulting in a 9 final tip radius tip of about $2 \mathrm{~nm}$, as judged from analysis of the indentation inside the 10 Lysenin rings. Amplitude modulation was used for imaging with free amplitude of $\sim 1.2 \mathrm{~nm}$ and operating set point amplitude of $\sim 0.9 \mathrm{~nm}$. Under these conditions we estimate the applied force following $F=\left(k_{c} / Q_{c}\right)^{*}\left(A_{0}\left(1-A_{s} / A_{0}\right)+h_{0} \sin (\theta / 2)\right.$ where $A_{0}$ is the free amplitude, $A s$ is the setpoint amplitude, $h_{0}$ is the step height of the sample, and $\theta$ is the phase delay of the feedback. Under our imaging conditions $F=44 \mathrm{pN} .{ }^{37}$ All experiments were performed at room temperature and in physiological buffer.

\section{High-speed atomic force microscopy (HS-AFM) image treatment}

Image treatment was limited to the correction of a first-order XY plane fit and XY drift correction of the HS-AFM movie. ${ }^{38}$

\section{High-speed atomic force microscopy (HS-AFM) data analysis}

The HS-AFM movie is considered a four dimensional matrix with lateral dimensions $X$ and $Y$, height dimension $Z$, and a time $t$. Time is subdivided in time-intervals $\tau$, the shortest $\tau$ is the time passing between the acquisitions of two subsequent frames. From this matrix, the height changes $\Delta z$ as a function of varying lag-time $\tau$, was calculated by subtraction, following $\Delta z_{i}(t, \tau)=z_{i}(t+\tau)-z_{i}(t)$ on each pixel. Socalled van Hove plots, i.e., the histogram distribution of the height variations was calculated for each pixel. Following, the shape of the van Hove plots for each $\tau$ was analysed according two parameters: Variance $V$ and Kurtosis $K$. While the variance informs about the width of the van Hove plots, hence about the intensity of motion, the Kurtosis reports about the non-Gaussianity, hence about non-Brownian behaviour. Each of these steps is performed for every lag-time $\tau$ and for every pixel. This data treatment resulted in variance and Kurtosis maps as shown in figure 2. Variance and Kurtosis were calculated using pre-built functions in Matlab(Matlab, Mathworks, Natick, USA).

To determine the local density, Voronoi tessellation was calculated from the localization of all particles. In order to determine the localization of all molecules in all frames, cross-correlation searches between a 360-fold symmetrized Lysenin ring (artificial reference) and each movie frame was performed. This resulted in crosscorrelation maps of each frame that featured about 700 cross-correlation peaks each. Peak searches allowed the localization of about 740000 molecules in the movie (about 700 in each frame). A lab-developed package ${ }^{18,38}$ integrated in ImageJ was used for the cross-correlation analysis and trajectory extraction. Using the particle localizations, Voronoi tessellation was calculated using a pre-built function in Matlab(Matlab, Mathworks, Natick, USA). 
1 Combining, the two above-described analysis allowed correlating variance and Kurtosis

2 with local protein density and evaluating how diffusion properties scale as a function

3 of membrane structure.

4 The area fraction $\phi$ was calculated taking in to account that the area occupied by a 5 Lysenin ring is $A_{(\mathrm{mol})}=\pi d / 4=113 \mathrm{~nm}^{2}$ (where $\mathrm{d}$ is the diameter of the lysenin ring, 6 i.e. center-to-center distance in the crystal packing), and the unit cell area of the 7 hexagonal close packing is $A_{\text {(unit cell) }}=2(\sqrt{3} / 4 a b)=124.7 \mathrm{~nm}^{2}$, resulting in $8 \phi=0.906899$. For our analysis $\phi$ is the ratio between $\mathrm{A}(\mathrm{mol})$ and the unit cell orVoronoi 9 cell in the real HS-AFM movie, in which it is located.

\section{Samplepreparation for transmission electron microscopy (TEM)observation}

Adsorption and oligomerization of Lysenin on asphingomyelin-containing lipid monolayer were performed incustum-designed Teflon wells of $4 \mathrm{~mm}$ in diameter and $1 \mathrm{~mm}$ in depth. $0.5 \mu \mathrm{l}$ of lipid solution (Sphingomyelin/Phophatidylcholin 1:4, Avanti Polar Lipids, Alabama, USA) at $0.1 \mathrm{mg} / \mathrm{ml}$ in chloroform were spread on $15 \mu$ l of Lysenin at $50 \mu \mathrm{g} / \mathrm{ml}$ and incubated for 1 hour at room temperature to reconstitute oligomers or overnight to form 2D-crystal patches. The interfacial surface formed by the lipid monolayer and the adsorbed protein was transferred to carbon-coated grids and analyzed by transmission electron microscopy.

Negative stain transmission electron microscopy (TEM) and image processing of single Lysenin oligomers

For imaging of negatively stained samples, the grid was washed with three droplets of pure water and subsequently negatively stained with $2 \%(\mathrm{w} / \mathrm{v})$ uranyl-acetate. The prepared grids were imaged using a Philips CM10 TEM (FEI Company, Eindhoven, the Netherlands) operated at $80 \mathrm{kV}$. The images were recorded by the $2 \mathrm{k} \times 2 \mathrm{k}$ sidemounted Veleta CCD camera (Olympus, Germany) at magnification of $130000 \mathrm{x}$. Under these conditions the pixel size at the sample level is $3.7 \AA$. Image processing was achieved with EMAN2 software package. ${ }^{39}$ The images were CTF (contrast transfer function) corrected and the particles were semi-automatically selected. The 'e2refine $2 d$ ' program was used to classify the particles, and produce reference-free class averages. The most populated class represented the top view.

\section{Cryo Transmission electron microscopy (cryo-TEM) and image processing of 2-crystal} patches of Lysenin

36 For cryo-TEM, the grid was blotted with Whatman filter paper and vitrified through plunging it into liquid nitrogen cooled liquid ethane using a vitrobot (FEI company, Netherlands). Frozen grids were transferred into a Philips CM200-FEG electron microscope using a Gatan 626 cryo-holder. Electron micrographs were recorded at an accelerating voltage of $200 \mathrm{kV}$ and a nominal magnification of $50000 \mathrm{x}$, using a lowdose system $\left(10 \mathrm{e}^{-} / \AA^{2}\right)$ and keeping the sample at liquid nitrogen temperatures. 
1 Defocus values were around $-2.5 \mu \mathrm{m}$. Micrographs were recorded on a $4 \mathrm{~K} \times 4 \mathrm{~K}$ CMOS 2 camera (TVIPS, Germany). The pixel size at the sample level is $2.1 \AA$. The 2D-crystal 3 images were selected based on the presence of diffraction patterns with well-defined 4 spots and further treated using the $2 \mathrm{dx}$ software. ${ }^{40}$

5

6 ASSOCIATED CONTENTS

7

\section{Supporting Information}

9 The Supporting Information is available free of charge on theACS Publications

10 websiteat DOI:

11

\section{ACKNOWLEDGEMENTS}

13 The authors thank Drs. Timo Betz, Pierre Sens, Didier Marguet andSebastienMailfert 14 for important discussions about thedata analysis. This work was funded by the ANR 15 grant financing the A*MIDEX program (ANR-11-IDEX-0001-02) and a European 16 Research Council (ERC) Consolidator Grant (\#310080). 


\section{REFERENCES}

1. Engelman, D. M. Membranes Are More Mosaic Than Fluid. Nature2005, 438, 578-580.

2. Fujiwara, T. Phospholipids Undergo Hop Diffusion in Compartmentalized Cell Membrane. The J Cell Biol2002, 157, 1071-1082.

3. Korlach, J.; Schwille, P.; Webb, W. W.; Feigenson, G. W. Characterization of Lipid Bilayer Phases by Confocal Microscopy and Fluorescence Correlation Spectroscopy. PNAS1999, 96, 8461-8466.

4. Simons, K.; Gerl, M. J. Revitalizing Membrane Rafts: New Tools and Insights. Nat RevMol Cell Biol2010, 11, 688-699.

5. Dietrich, C.; Yang, B.; Fujiwara, T.; Kusumi, A.; Jacobson, K. Relationship of Lipid Rafts to Transient Confinement Zones Detected by Single Particle Tracking. BiophJ2002, 82, 274-284.

6. Marguet, D.; Lenne, P.-F.; Rigneault, H.; He, H.-T. Dynamics in the Plasma Membrane: How to Combine Fluidity and Order. EMBO J2006, 25, 3446-3457.

7. Höfling, F.; Franosch, T. Anomalous Transport in the Crowded World of Biological Cells. Rep Prog Phys2013, 76, 046602.

8. Krapf, D. Chapter Five - Mechanisms Underlying Anomalous Diffusion in the Plasma Membrane. In Current Topics in Membranes, Anne, K. K., Ed. Academic Press: 2015; Vol. Volume 75, pp 167207.

9. Metzler, R.; Jeon, J.-H.; Cherstvy, A. G.; Barkai, E. Anomalous Diffusion Models and Their Properties: Non-Stationarity, Non-Ergodicity, and Ageing at the Centenary of Single Particle Tracking. Phys Chem Chem Phys2014, 16, 24128-24164.

10. Sekizawa, Y.; Hagiwara, K.; Nakajima, T.; Kobayashi, H. A Novel Protein, Lysenin, That Causes Contraction of the Isolated Rat Aorta : Its Puriification from the Coleomic Fluid of the Earthworm, Eisenia Foetida. BiomedRes1996, 17, 197-203.

11. De Colibus, L.; Sonnen, Andreas F. P.; Morris, Keith J.; Siebert, C. A.; Abrusci, P.; Plitzko, J.; Hodnik, V.; Leippe, M.; Volpi, E.; Anderluh, G.; Gilbert, Robert J. C. Structures of Lysenin Reveal a Shared Evolutionary Origin for Pore-Forming Proteins and Its Mode of Sphingomyelin Recognition. Structure2012, 20, 1498-1507.

12. lacovache, I.; van der Goot, F. G.; Pernot, L. Pore Formation: An Ancient yet Complex Form of Attack. Biochim Biophys Acta - Biomemb2008, 1778, 1611-1623.

13. Yilmaz, N.; Yamada, T.; Greimel, P.; Uchihashi, T.; Ando, T.; Kobayashi, T. Real-Time Visualization of Assembling of a Sphingomyelin-Specific Toxin on Planar Lipid Membranes. Bioph J2013, 105, 1397-1405.

14. Yilmaz, N.; Kobayashi, T. Visualization of Lipid Membrane Reorganization Induced by a PoreForming Toxin Using High-Speed Atomic Force Microscopy. ACS Nano2015, 9, 7960-7967.

15. Dahmane, S.; Rubinstein, E.; Milhiet, P.-E. Viruses and Tetraspanins: Lessons from Single Molecule Approaches. Viruses2014, 6, 1992-2011.

16. Ritchie, K.; Shan, X.-Y.; Kondo, J.; Iwasawa, K.; Fujiwara, T.; Kusumi, A. Detection of NonBrownian Diffusion in the Cell Membrane in Single Molecule Tracking. Bioph J2005, 88, 22662277.

17. Ando, T.; Kodera, N.; Takai, E.; Maruyama, D.; Saito, K.; Toda, A. A High-Speed Atomic Force Microscope for Studying Biological Macromolecules. PNAS2001, 98, 12468-12472.

18. Casuso, I.; Khao, J.; Chami, M.; Paul-Gilloteaux, P.; Husain, M.; Duneau, J.-P.; Stahlberg, H.; Sturgis, J. N.; Scheuring, S. Characterization of the Motion of Membrane Proteins Using HighSpeed Atomic Force Microscopy. Nat Nanotechnol2012, 7, 525-529.

19. Fujiwara, T.; Ritchie, K.; Murakoshi, H.; Jacobson, K.; Kusumi, A. Phospholipids Undergo Hop Diffusion in Compartmentalized Cell Membrane. JCell Biol2002, 157, 1071-1082.

20. García-Sáez, A. J.; Schwille, P. Fluorescence Correlation Spectroscopy for the Study of Membrane Dynamics and Protein/Lipid Interactions. Methods2008, 46, 116-122.

21. Kegel, W. K.; Blaaderen; Alfons, v. Direct Observation of Dynamical Heterogeneities in Colloidal Hard-Sphere Suspensions. Science2000, 287, 290-293.

22. Valentine, M. T.; Kaplan, P. D.; Thota, D.; Crocker, J. C.; Gisler, T.; Prud’homme, R. K.; Beck, M.; Weitz, D. A. Investigating the Microenvironments of Inhomogeneous Soft Materials with Multiple Particle Tracking. Phys Rev E2001, 64.

23. Marcus, A. H.; Schofield, J.; Rice, S. A. Experimental Observations of Non-Gaussian Behavior and 
Stringlike Cooperative Dynamics in Concentrated Quasi-Two-Dimensional Colloidal Liquids. Phys Rev E1999, 60, 5725.

24. Berthier, L.; Biroli, G. Theoretical Perspective on the Glass Transition and Amorphous Materials. Rev Mod Phys2011, 83, 587-645.

25. Poupon, A. Voronoi and Voronoi-Related Tessellations in Studies of Protein Structure and Interaction. Curr Opin Struct Biol2004, 14, 233-241.

26. Reis, P. M.; Ingale, R. A.; Shattuck, M. D. Crystallization of a Quasi-Two-Dimensional Granular Fluid. Phys Rev Lett2006, 96, 258001.

27. Zhao, K.; Bruinsma, R.; Mason, T. G. Local Chiral Symmetry Breaking in Triatic Liquid Crystals. Nat Commun2012, 3, 801.

28. Sciortino, F.; Tartaglia, P.; Zaccarelli, E. Evidence of a Higher-Order Singularity in Dense ShortRanged Attractive Colloids. Phys Rev Lett2003, 91, 268301.

29. Fehr, T.; H.Lowen. Glass Transition in Confined Geometry. Physical Review E: 1995.

30. Mayer, C.; Zaccarelli, E.; Stiakakis, E.; Likos, C. N.; Sciortino, F.; Munam, A.; Gauthier, M.; Hadjichristidis, N.; latrou, H.; Tartaglia, P.; Löwen, H.; Vlassopoulos, D. Asymmetric Caging in Soft Colloidal Mixtures. Nat Mat2008, 7, 780-784.

31. Scheuring, S.; Sturgis J. N. Chromatic Adaptation of Photosynthetic Membranes. Science2005, 309, 484-487.

32. Fotiadis, D.; Liang, Y.; Filipek, S.; Saperstein, D. A.; Engel, A.; Palczewski, K. Atomic-Force Microscopy: Rhodopsin Dimers in Native Disc Membranes. Nature2003, 421, 127-128.

33. Buzhynskyy, N.; Salesse, C.; Scheuring, S. Rhodopsin Is Spatially Heterogeneously Distributed in Rod Outer Segment Disk Membranes. JMol Rec2011, 24, 483-489.

34. Minton, A. P. Lateral Diffusion of Membrane Proteins in Protein-Rich Membranes. BiophJ1989, 55, 805-808.

35. Angelova, M. I.; Dimitrov, D. S. Liposome Electroformation. Faraday Discuss Chem Soc1986, 81, 303-311.

36. Colom, A.; Casuso, I.; Rico, F.; Scheuring, S. A Hybrid High-Speed Atomic Force-Optical Microscope for Visualizing Single Membrane Proteins on Eukaryotic Cells. Nat Commun2013, 4.

37. Ando, T. High-Speed Atomic Force Microscopy Coming of Age. Nanotechno/2012, 23, 062001.

38. Husain, M.; Boudier, T.; Paul-Gilloteaux, P.; Casuso, I.; Scheuring, S. Software for Drift Compensation, Particle Tracking and Particle Analysis of High-Speed Atomic Force Microscopy Image Series: Software for High-Speed Atomic Force Microscopy Image Series. JMol Rec2012, 25, 292-298.

39. Ludtke, S. J.; Baldwin, P. R.; Chiu, W. Eman: Semiautomated Software for High-Resolution Single-Particle Reconstructions. J Struct Biol1999, 128, 82-97.

40. Scherer, S.; Kowal, J.; Chami, M.; Dandey, V.; Arheit, M.; Ringler, P.; Stahlberg, H. 2dx_Automator: Implementation of a Semiautomatic High-Throughput High-Resolution CryoElectron Crystallography Pipeline. J Struct Bio/2014, 186, 302-307. 

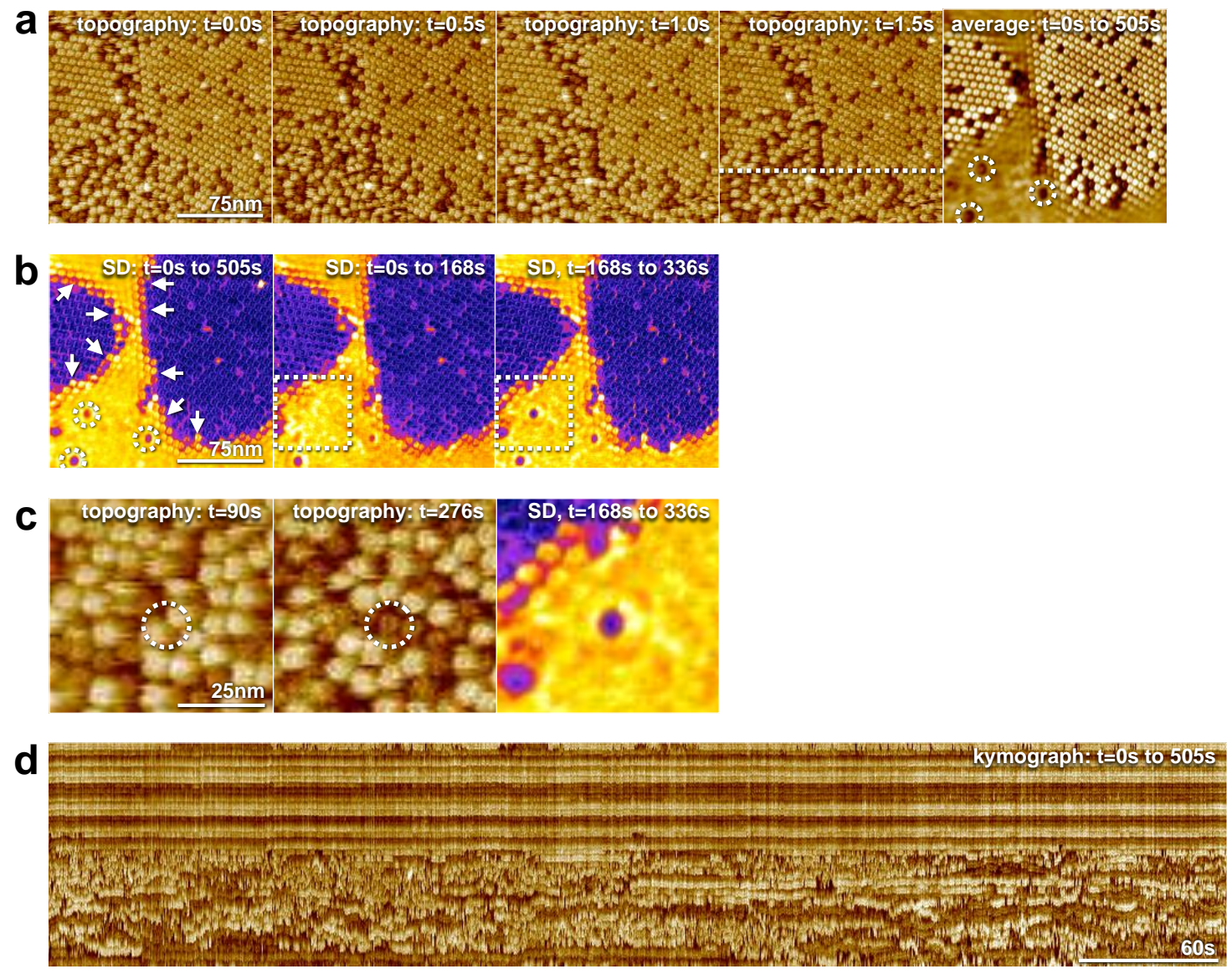

Figure 1) Lysenindynamics is location dependent. a) Four left panels: HS-AFM movie frames (Supplementary Movie 1) ofLysenin inasphingomyelin/cholesterol (1:1) bilayer. Right panel:Timeaveraged frame displaying the positional stability and the high mobility of proteins in the solid and fluid domains(full false color scale: $10 \mathrm{~nm}$ ). b) Left: standard deviation (SD) map of the pixel height values $(t=0-505 s)$. Lattice borders with well-defined molecular positions of varying SD(arrows). SD maps $(t=0-$ $168 \mathrm{~s}$, center) and $(\mathrm{t}=168-336 \mathrm{~s}$, right): At $\mathrm{t}=168 \mathrm{~s}$ alocation(dashed squares)drastically changed dynamics(full false color scale: $0.3<S D<1.6 \mathrm{~nm}$ ).c) Individual frames $\mathrm{t}=90 \mathrm{~s}$ and $\mathrm{t}=276 \mathrm{~s}$ of the molecular organization corresponding to the outlines inb), see also supplementary movie 2 . Right: SD map ( $t=168$ 336s) of this membrane region displaying the annular alteration of diffusion dynamics around the stuck molecule. d) Kymograph (of thewhite dashed line in a). Stable (top), highly mobile (bottom), and molecules switching between stability andhigh mobility (middle) are visible during the entire movie. 


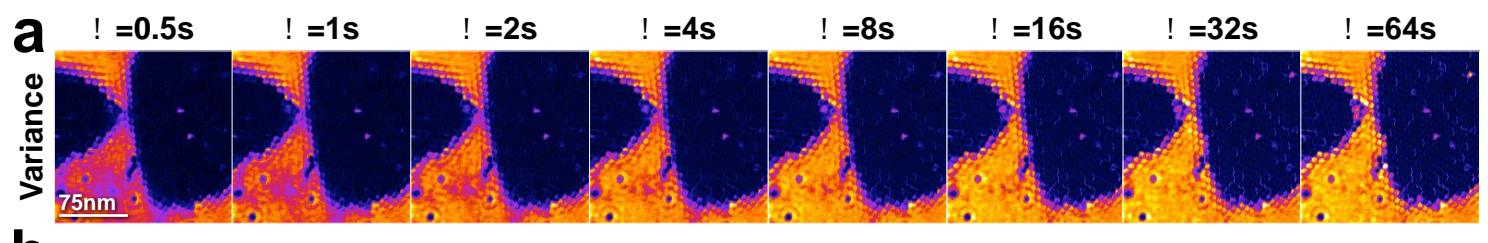

b

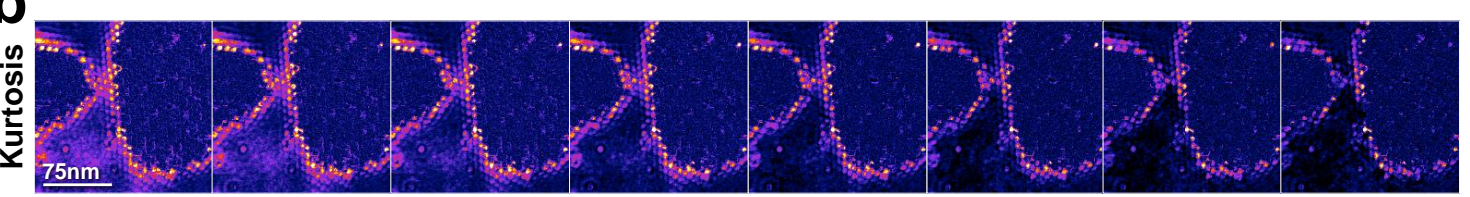

2 Figure 2) Detection of areas of non-Brownian dynamics. a) Variance(V)of the distribution of height 3 changes (false color scale: $\left.0<V<2 \mathrm{~nm}^{2}\right)$.b) Kurtosis(K)(non-Gaussianity) of the distribution of the height 4 changes (false color scale: $2.5<K<5.0)$ ). 

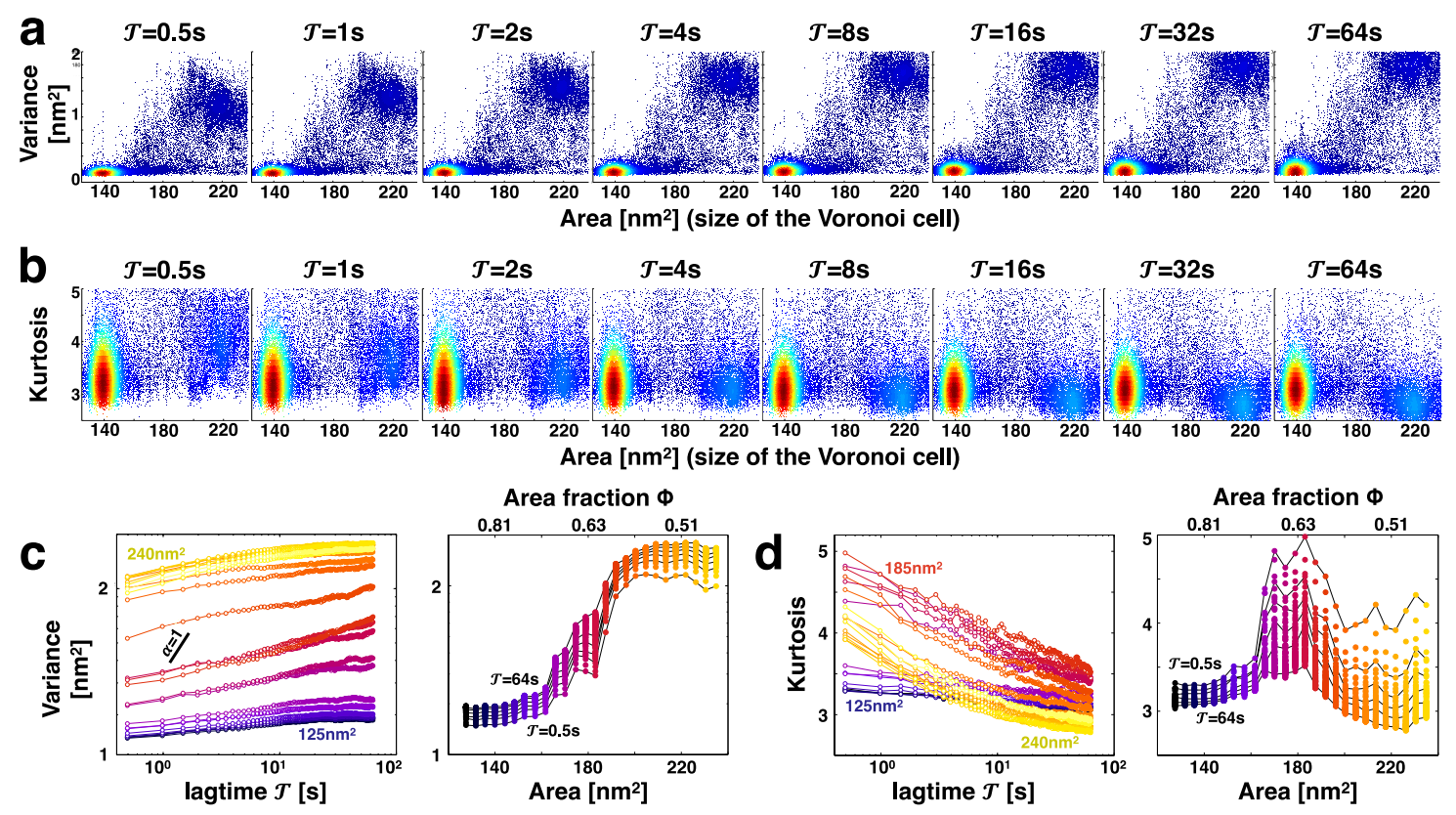

Figure 3) Diffusion as function of local density. a)Distribution of Variance and b) Kurtosis for all pixels as a function of Voronoi cell area and lag time (color indicates the concentration of data points in the plot area from blue (low abundance) to red (high abundance)). c) Variance and d) Kurtosis as function of lag time and Voronoi cell area: Each point is the median of all pixels with the same characteristics. 